\title{
Linear polymers with competing interactions: Swollen linear, swollen branched, and compact scaling regimes
}

\author{
R. Dekeyser \\ Instituut voor Theoretische Fysica, Katholieke Universiteit Leuven, B-3001 Heverlee, Belgium \\ E. Orlandini \\ Department of Physics, Theoretical Physics, University of Oxford, Oxford OX1 3NP, England \\ A.L. Stella \\ Istituto Nazionale di Fisica della Materia, Dipartimento di Fisica e Sezione, \\ Istituto Nazionale di Fisica Nucleare, Università di Padova, I-35131 Padova, Italy \\ M.C. Tesi \\ Mathematical Institute, University of Oxford, Oxford OX1 3NP, England
}

(Received 6 April 1995)

\begin{abstract}
Models of linear polymers with competing interactions favoring ramification into thin branches on one hand, and compactification on the other hand, are shown to possess a phase diagram with swollen linear, swollen branched, and compact regimes. Between each pair of these regimes, a multicritical transition occurs. The three transitions merge in a single, very unstable multicritical point. Evidence of such behavior is obtained by renormalization methods applied exactly to a model on a hierarchical lattice, and approximately to a system on a square lattice. The hierarchical model calculations, discussed in much detail, are particularly extensive and give the advantage of an almost complete characterization of the different scaling behaviors, exact at least within the limits of numerical accuracy. The strong qualitative evidence from renormalization group approaches is further confirmed by an extensive exact enumeration analysis for a third model on the square lattice. The scalings at various multicritical transitions are studied in detail and universality issues are discussed.
\end{abstract}

PACS number(s): 61.41.+e, 64.60.Ak, 64.60.Cn, 64.60.Kw

\section{INTRODUCTION}

Both chainlike and branched polymers behave at high temperatures in good solvents as swollen fractal structures, in their self-avoiding walk (SAW) and branched polymer (BP) regimes, respectively. This is reflected by a value $\nu>1 / d$ for the exponent in the law $R \propto N^{\nu}$, connecting the average linear size $R$ to the total "length" $N$ (the molecular weight). As the temperature is reduced, a collapse transition is expected to occur in these systems at a certain $\Theta$ temperature. This collapse is induced by van der Waals attractions between the monomers. Below the $\Theta$ temperature, the polymer behaves as a compact object $(\nu=1 / d)$ with the same finite density at all length scales, in the limit of infinite molecular weight. This is called the compact phase (CP).

These $\Theta$ transitions were the object of many investigations in recent years [1-12], mostly in connection with universality issues. Indeed, whereas the universality of the $\nu$ exponents for the swollen (high $T$ ) and the collapsed (low $T$ ) phases is both theoretically and numerically well supported, difficulties in the determination of the exponents at the $\Theta$ point, like $\nu_{\theta}$, make the existence of a unique multicritical universality class more controversial. Moreover, in the case of branched polymers, there exist models [10-12] displaying clearly two distinct lines of $\Theta$ collapses, most likely belonging to different universality classes.

Until now, much less attention has been devoted to the possibility that, due to peculiar interaction mechanisms, collapses could occur with some intermediate scaling regime separating, in a whole interval of temperatures, the fully swollen from the compact phases. Another case is that of a "collapse" in which the attractive interactions cause a transition to a low temperature regime with higher fractal dimension (lower $\nu$, but still $>1 / d)$, which is never followed by a complete collapse into a constant density regime.

The possibility that such type of scenarios could replace, under suitable conditions, the standard picture of collapse implies a more substantial degree of nonuniversality than contemplated in the usual $\Theta$-point statistical mechanics. The fact that modified interaction mechanisms can alter the standard "phase diagram" of an interacting polymer system should also be more easily detectable at the experimental level.

In a recent Letter [13], it was proposed that, for certain models of interacting linear polymers, the intermediate, partially collapsed phase should be identified with the swollen regime of branched polymers, which have a higher fractal dimension than self-repelling chains. The mechanism leading to a partially collapsed intermediate 
branched polymer regime is connected with the presence in the Hamiltonian of at least two competing interactions: one favoring collapse (an attractive force), the other representing some repulsion between different branches of the collapsed structure (this force counterbalances the previous attractive one). A collapse from linear to branched polymer behavior was clearly obtained in Ref. [13] for a model defined on a hierarchical lattice. Convincing evidence of a collapse into a swollen branched polymer regime was also given by a series analysis of a linear model on a two-dimensional (2D) Euclidean lattice [13], which was not supposed to possess a CP. Unfortunately, the existence of a further collapse transition from branched polymer to compact regime, claimed in Ref. [13] for the hierarchical model, was revealed to be spurious and had to be excluded on the basis of a much more accurate numerical analysis. So, at the present stage, both the exact and approximate model calculations reported in Ref. [13] materialize only the second scenario outlined above, in which the collapse from linear to branched polymer regimes is never followed by a further collapse into a CP.

Some of the ideas of Ref. [13] were further developed by Bradley [14], who considered the phenomenon of a partial SAW-BP collapse within the framework of the vacancy mediated interactions for self-avoiding vesicles on the 2D hexagonal lattice. For the model of Ref. [14], a collapse from BP to CP is indeed taking place. However, in this context, the mechanism leading to the intermediate phase is an asymmetry between the interior and the exterior of the vesicle. While this could be plausible for some polymer ring problems, such a model does not seem meaningful for open chains. In addition, this approach does not throw light on the multicritical scaling properties and on the flow of the model parameters under renormalization.

In this work we reconsider a basic suggestion of Ref. [13], that the BP regime can occur as intermediate between the swollen linear and compact ones, and we show that such a possibility is indeed realized in models with suitable competing interactions.

The possibility for a linear polymer system that an intermediate BP regime is realized besides the swollen and the collapsed ones, suggests that one should look for a rich phase diagram, displaying lines (or surfaces) of transitions between each pair of phases, and a multicritical point where these lines meet. A further intriguing feature to check is whether there could exist more than a single compact regime for such systems. Such a feature has recently been debated [9-12] for models of interacting lattice animals and branched polymers.

The purpose of the present paper is to discuss in detail different models and approaches that allow a description of such phase diagrams, and of the associated critical and multicritical scaling properties. On one hand, the variety of models in which we recognize the basic phenomena outlined above is an indication that these phenomena are not so exceptional as one could suspect at first sight. At the same time, the different models are chosen to make the application possible of methods with varying rigor and power.
In the first section, we consider a model defined on a finitely ramified hierarchical lattice (the 3D Sierpinski gasket) and present the results of an extensive investigation of exact renormalization group recursions for it. In view of the large size of such recursions and the difficulty of numerically determining fixed point solutions and their stability, particular care must be used in the analysis. Our discussion tries to present in a very explicit and clear way the various difficulties and their solutions. Hopefully it will also be of methodological value for readers who want to embark on similar extensive calculations. As already mentioned above, in Ref. [13] results for a similar, less complicated model on the two-dimensional gasket were presented. In that case only one interaction parameter was present and no complete phase diagram in the sense discussed above could be obtained. Due to insufficient numerical precision in the analysis, a collapse from BP to the compact regime was erroneously predicted to follow the SAW-BP partial collapse at very high values of the attractive interactions. The results of the first section also supply a valid illustration of some of the collapse properties which were incorrectly attributed to the model of Ref. [13].

In spite of the partly artificial character of models defined on hierarchical lattices, these can provide, through exact calculations, a correct qualitative description of phenomena occurring on Euclidean lattices for more realistic models, especially in the case of polymer systems.

In Sec. II, we describe an approximate real space renormalization group (RSRG) approach to multiple scaling regimes in a model on a $2 \mathrm{D}$ Euclidean lattice. Besides confirming the physical picture extracted in Sec. I in a relatively more simple, though approximate technical framework, this approach gives a further example of how competing interactions, inducing intermediate collapse, can be cast within a model.

A third example is finally considered in Sec. III, where this time results of an extensive analysis of exact enumerations on the square lattice are reported for a different model, containing nevertheless the same characteristics of two competing interactions. Some concluding remarks are made in Sec. IV.

\section{A MODEL ON A 3D FRACTAL LATTICE}

\section{A. Introduction}

There are many motivations for studying problems of polymer statistics on fractal lattices. Fractal lattices are somehow intermediate between periodical and disordered ones, and the study of critical phenomena occurring on them is interesting in its own right [15].

The connectivities of fractal lattices are simple enough to allow often an exact renormalization treatment exploiting their hierarchical and scale invariant structure. Especially in the case of polymer problems, the approaches on hierarchical lattices have been revealed to be useful, because, in contrast with, e.g., spin models, nontrivial critical thresholds can be obtained. Most im- 
portant for us here, both for linear and branched selfattracting polymers, models on fractal lattices have even shown the occurrence of standard $\Theta$ transitions, of the type commonly studied on regular lattices. In these transitions the $\nu$ exponent remains at the value appropriate to the swollen regime for all $T>T_{\theta}$, whereas $\nu=1 / \bar{d}$ for $T<T_{\theta}, \bar{d}$ being the fractal lattice dimension. At $T=T_{\theta}$, the tricritical point, $\nu=\nu_{\theta}$, with $\nu_{\theta}$ different from both previous values.

As already mentioned in the Introduction, in a previous publication [13] a polymer was modeled by a static one-tolerant trail [16] on a 2D Sierpinski gasket. Since in such a lattice twice visited sites are allowed for this trail (but not twice visited bonds), an attractive (negative) energy was introduced, favoring such double sites. Upon increasing this energy (or lowering the temperature, which is the same in this case), one can only find a single collapse from the swollen SAW to a swollen BP regime. A natural choice in order to enrich the phase diagram is to ntroduce an extra interaction energy, favoring nearest neighbor visited sites not directly connected by chain steps. If we assume in the one-tolerant trail that the double site energy becomes repulsive and grows to $+\infty$, we are left with a strictly self-repelling chain with nearest neighbor attractive interactions. We know that this model, on a 2D Sierpinski gasket, does not lead to a standard $\Theta$ transition from SAW to CP, as normally expected. So, while the above two interactions in the trail can have competitive effects in the sense discussed in the Introduction (enhancing the twice visited sites ends up in reducing the number of nearest neighbor pairs of not consecutively visited sites), the phase diagram to be expected on the 2D Sierpinski gasket is not satisfactory enough for us, because it lacks as a basic ingredient the standard linear polymer $\Theta$ transition.

In order to introduce this feature, the solution is to upgrade the gasket to a $3 \mathrm{D}$ one. In this case we know that the self-repelling chain has a standard $\Theta$ transition and we can expect interesting effects to be introduced as soon as we start decreasing from $+\infty$ the interaction energy for twice visited sites.

\section{B. Model and method}

The model that we study here is somewhat different from those commonly used in order to model linear [17] and branched [18] polymers on fractals, both by the geometrical restrictions imposed on the walks and by the kind of attractions considered. More precisely, we impose that the edges of the gasket are visited at most once and sites at most twice. A walk which can visit a bond at most once is called in the literature a one-tolerant trail. Here we add an extra restriction on the site visitations. It should be noted here that the restriction that we impose on the sites of the gasket is not a direct consequence of the constraint on the edges. In fact, thanks to the ramification number of the 3D Sierpinski gasket, a site could also be visited three times without breaking the edge constraint. We have made this choice for two reasons: first, we have with this restriction a natural generalization of the two-dimensional model mentioned before [13]; second, without this restriction we would have to manage an enormous number of partial generating functions in the RG treatment. As a matter of fact, the situation is just difficult enough with the 19 functions we have to consider with our choice, as will become clear in the following. In order to simplify the exact treatment, without making the physics poorer, we impose an additional geometrical constraint on each internal vertex by imposing that the walk has always to move out of the elementary up-tetrahedron visited in the last step. In this way we realize recursions analogous to those valid on a, socalled, four-simplex lattice [19]. The simplex character of the recursions again makes the numerical problem more tractable. The problem we consider is static, so, in spite of the terminology, we actually consider as independent walk configurations the silhouettes of a class of dynamical one-tolerant trails on the gasket. Concerning the kind of interactions considered, in addition to the standard monomer-monomer interaction introduced by assigning an energy $\epsilon_{i}\left(\epsilon_{i}<0\right)$ to each pair of nearest neighbor nonconsecutively visited sites, we weight with an energy $\epsilon_{d}\left(\epsilon_{d}<0\right)$ each site in which a self-crossing of the walk occurs.

By switching on only the $\epsilon_{i}$ interaction, we expect to model the standard situation of a linear polymer in a bad solvent. Indeed, a chain with a large scale exclusion and attractive interactions is known to undergo a $\Theta$ transition from the SAW to a compact regime [17], when embedded in a 3D Sierpinski gasket. On the other hand, if the twice-visited site attraction is dominant, we will observe numerically two consecutive transitions taking place [13]: the first one is from a linear to a branched polymer behavior followed by a further collapse from the swollen branched regime into a compact regime. When the two interactions compete, we expect that the multicritical lines will converge in a supermulticritical point.

In a grand canonical description of the model we introduce a step fugacity $x$, a fugacity $v=\exp \left(-\epsilon_{i} / k T\right)$ conjugate to the number of monomer-monomer interactions, and a fugacity $u=\exp \left(-\epsilon_{d} / k T\right)$ associated with each twice visited site. The generating function, normalized per lattice site, can be written as

$$
\begin{aligned}
\mathcal{Z}(x, v, u) & =\sum_{\text {walks }} x^{N} v^{N_{i}} u^{N_{d}} \\
& =\sum_{N, N_{i}, N_{d}} C\left(N, N_{i}, N_{d}\right) x^{N} v^{N_{i}} u^{N_{d}} \\
& =\sum_{N} Z_{N}(u, v) x^{N}
\end{aligned}
$$

where $C\left(N, N_{i}, N_{d}\right)$ represents the number of configurations of $N$-step trails with $N_{i}$ nearest neighbor pairs and $N_{d}$ self-crossings, normalized per lattice site, and $Z_{N}$ is the canonical partition function for a walk of $N$ steps.

An average end-to-end distance can be defined as

$$
\begin{aligned}
R(x, u, v)= & \mathcal{Z}^{-1}(x, u, v) \sum_{N, N_{i}, N_{d}} R\left(N, N_{i}, N_{d}\right) \\
& \times C\left(N, N_{i}, N_{d}\right) x^{N v^{N_{i}} u^{N_{d}}}
\end{aligned}
$$


where $R\left(N, N_{i}, N_{d}\right)$ is the average end-to-end distance for walks with given $N, N_{i}$, and $N_{d}$. In analogy with the behavior on Euclidean lattices, we can expect that $Z_{N}(u, v) \propto \mu(u, v)^{N} N^{-\theta}$ for $N \rightarrow \infty$, where $\theta$ is the entropic exponent. The critical surface is given by $x_{c}=$ $x_{c}(u, v) \equiv \mu^{-1}(u, v)$, and for $x \rightarrow x_{c}^{-}$

$$
\mathcal{Z}(x, u, v) \sim\left[x_{c}(u, v)-x\right]^{1-\theta}
$$

and

$$
R(x, u, v) \sim\left[x_{c}(u, v)-x\right]^{-\nu}
$$

if

$$
\begin{aligned}
\langle R\rangle_{N}(u, v) & =\frac{\sum_{N_{i}, N_{d}} R\left(N, N_{i}, N_{d}\right) C\left(N, N_{i}, N_{d}\right) v^{N_{i}} u^{N_{d}}}{\sum_{N_{i}, N_{d}} C\left(N, N_{i}, N_{d}\right) v^{N_{i}} u^{N_{d}}} \\
& \sim N^{\nu} \quad \text { for } N \rightarrow \infty .
\end{aligned}
$$

For quantities like $\mathcal{Z}$, a normalization per lattice site is implicitly understood in the thermodynamic limit.

Exponents like $\theta$ and $\nu$ in Eqs. (1.3)-(1.5) will in general depend on the point at which the critical surface is approached. The renormalization group helps in determining all this. For simplicity, we discuss only the "thermal" exponents, like $\nu$. We thus set up an iterative scheme for calculating $\mathcal{Z}$ in Eq. (1.1), restricted to ringlike closed walks. With such a restriction, Eq. (1.3) is replaced by $\mathcal{Z}(x, u, v) \sim\left[x_{c}(u, v)-x\right]^{2-\alpha}$, where $\alpha=2-\bar{d} \nu$.

The knowledge of a certain number of partial generating functions $X_{n, j}$ for walks on a gasket of generation $n(j=1,2, \ldots, 19$, in our case $)$ allows one to determine $\mathcal{Z}_{n+1}$, the generating function (1.1) for the gasket of side $2^{n+1}$, as well as the same kind of partial generating functions for the $(n+1)$ th generation gasket, $X_{n+1, j}$. Figure 1 represents schematically all these 19 generating functions, each one with its specific restrictions on the walk, or walks, stepping on the gasket. Due to the large number of partial generating functions, the transformation

$$
X_{n+1, j}=F_{j}\left(\left\{X_{n, k}\right\}\right)
$$

can be determined only by exact enumeration techniques on the computer. An example of one of the many configurations that have to be counted in this process is given in Fig. 2. The set of nonlinear equations (1.6) is too large to be reported here, but it is available upon request [20]. One important simplifying feature is the fact that $x, u$ and $v$ do not explicitly appear in the recursions (1.6), but only enter the initial conditions of the various $X_{0, j}$. This feature is due to the simplex character of our recursions.

The equations (1.6) can be interpreted as a standard RG transformation, with rescaling $\ell=2$, in a 19dimensional parameter space. At a critical fixed point $X_{j}^{*}$ one defines a set of scaling fields $h_{i}$ with dimension $y_{i}$ and a quantity like the singular part $\mathcal{Z}_{s}$ of the generating function will transform as
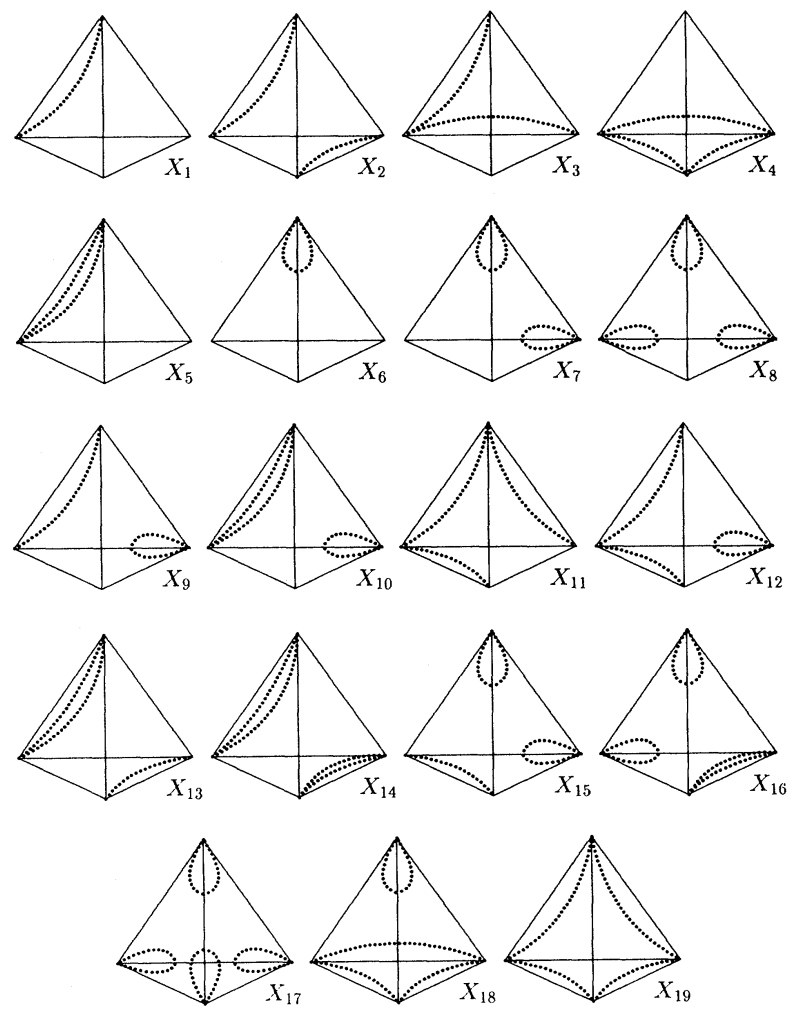

FIG. 1. Diagrams representing typical contributions in terms of walks for the 19 restricted generating functions.

$$
\mathcal{Z}_{s}\left(\left\{h_{i}\right\}\right)=\ell^{-\bar{d}} \mathcal{Z}_{s}\left(\left\{\ell^{y_{i}} h_{i}\right\}\right)
$$

where $y_{1}=1 / \nu>0$ is the only relevant eigenvalue. If the fixed point is tricritical, there will be an additional relevant field, i.e., $y_{2} \equiv \phi / \nu>0$. Since in the RG iteration the initial $X_{j}$ and $h_{i}$ are specified in terms of three fugacities, a critical fixed point singles out a critical surface $\left(h_{1}=0\right)$ in the $(x, u, v)$ space, while a tricritical fixed point singles out a line $\left(h_{1}=0, h_{2}=0\right)$. A last possibility is that of three relevant fields $\left(y_{1}>0, y_{2}>0, y_{3}>0\right)$, which singles out a fully unstable multicritical point on the surface.
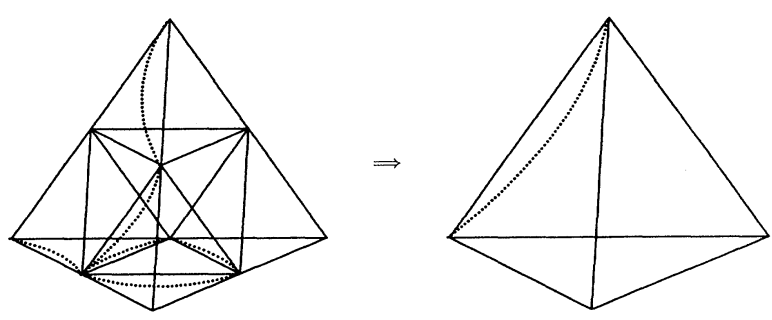

FIG. 2. Example of the renormalization procedure in the Sierpinski gasket. The graph on the left gives a contribution $X_{1}^{2} X_{3}^{2}$ to $X_{1}^{\prime}$. 


\section{Results}

For fixed $u$ and $v$, at $x=x_{c}(u, v)$ the thermodynamics of the model is determined by the fixed point reached by the RG recursions as the order of the iteration $n$ goes to infinity. The possible fixed points can be divided into two categories. In order to explain this, we have to give a few details about the recursion equations, which we rewrite symbolically as

$$
\vec{X}^{\prime}=\vec{F}(\vec{X})
$$

All 19 components of the vector $\vec{X}$ are non-negative weight factors and the 19 functions $X_{j}$ are all built up as a sum of positive contributions. The component $X_{6}$, related to the simple loop diagram in one corner of the tetrahedron, plays a peculiar role in these equations. Some of these equations read as follows:

$$
\begin{aligned}
X_{6}^{\prime} & =X_{6}+\cdots, \\
X_{7}^{\prime} & =X_{6}^{2}+\cdots, \\
X_{8}^{\prime} & =X_{6}^{3}+\cdots, \\
X_{17}^{\prime} & =X_{6}^{4}+\cdots
\end{aligned}
$$

From this, it is immediately clear that $X_{6}$ can never decrease under the renormalization flow. Three different situations can occur.

(1) If a finite fixed point $\vec{X}^{*}$ is encountered, it must mean that the remaining terms in the equation for $X_{6}^{\prime}$, represented with the ellipses, go to zero upon iteration. It turns out that in such a case also the terms corresponding to the ellipses in the other equations written above go to zero. In that case, however, $X_{6}^{*}$ can take an arbitrary value: this implies a marginal eigenvalue $(y=0)$ in the linearized renormalization equations around the fixed point, and there exists a line of fixed points. The particular choice of $X_{6}$ does not affect the critical exponents. Such a situation has been previously discussed in Ref. [13]. In this sense even the high temperature fixed point is a fixed line, where all $X_{j}$ are zero, except for the arbitrary $X_{6}$, with $X_{7}, X_{8}$, and $X_{17}$ its second, third, and fourth powers, respectively. Other nontrivial fixed points of this type have some fixed nonzero components, whereas other components may also be functions of the arbitrary $X_{6}$ (see Table I).

(2) A second possibility occurs in the case of a fixed point when in the above equations the remaining terms go to zero only for $X_{6}=0$. In such a case, we have a true isolated fixed point and not a fixed line. An example of such a point is found at $X_{19}^{*}=3^{-4 / 3}$, the remaining $X_{j}^{*}$ being zero. The sixth recursion equation looks more specifically as

$$
X_{6}^{\prime}=X_{6}+3 X_{6}^{3} X_{19}+\cdots
$$

(The terms that are left out become zero at this fixed point.) This recursion equation still leads for this fixed point to a marginal eigenvalue, corresponding to the $X_{6}$ direction, but the full nonlinear equation makes this direction marginally repulsive.

(3) If the ellipsis in the equation for $X_{6}^{\prime}$ does not vanish, $X_{6}$ is an ever increasing function of the renormalization flow, which finally goes to infinity. It is then convenient to rescale the other components by appropriate powers of $X_{6}$, such that the remaining equations become independent of $X_{6}$. We therefore define a vector $\vec{Y}$ by

$$
Y_{j}=X_{6}^{n_{j}} X_{j}
$$

TABLE I. Relevant fixed points of the recursion system for the model on the Sierpinski gasket. In the left part of the table, $E$ stands for an arbitrary value of $X_{6}$ and indicates a line of fixed points. The right hand part of the table gives fixed points for the rescaled variables.

\begin{tabular}{llcc|c|ccccc}
\hline & I & II & III & IV & V & \multicolumn{1}{c}{ VI } & VII & VIII & \\
& SAW & CP & SAW-CP & $T$ & BP & SAW-BP & BP-CP & S-BP-CP & \\
\hline$X_{1}$ & 0.429445 & 0 & $1 / 3$ & 0 & 0 & 0.429445 & 0 & $1 / 3$ & $Y_{1}$ \\
$X_{2}$ & 0.049984 & $22^{-1 / 3}$ & $1 / 3$ & 0 & 0 & 0.049984 & $22^{-1 / 3}$ & $1 / 3$ & $Y_{2}$ \\
$X_{3}$ & 0 & 0 & 0 & 0 & 0 & 0 & 0 & 0 & $Y_{3}$ \\
$X_{4}$ & 0 & 0 & 0 & 0 & 0.054986 & 0.054986 & 0.054986 & 0.054986 & $Y_{4}$ \\
$X_{5}$ & 0 & 0 & 0 & 0 & 0.152751 & 0.152751 & 0.152751 & 0.152751 & $Y_{5}$ \\
$X_{6}$ & $E$ & $E$ & $E$ & 0 & 2.0785 & 2.0785 & 2.0785 & 2.0785 & $X_{6}^{\prime} / X_{6}$ \\
$X_{7}$ & $E^{2}$ & $E^{2}$ & $E^{2}$ & 0 & 0.661873 & 0.661873 & 0.661873 & 0.661873 & $Y_{7}$ \\
$X_{8}$ & $E^{3}$ & $E^{3}$ & $E^{3}$ & 0 & 0.372061 & 0.372061 & 0.372061 & 0.372061 & $Y_{8}$ \\
$X_{9}$ & $f(E)$ & 0 & $\tilde{f}(E)$ & 0 & 0 & 0.22109 & 0 & 0.130412 & $Y_{9}$ \\
$X_{10}$ & 0 & 0 & 0 & 0 & 0.061444 & 0.061444 & 0.061444 & 0.061444 & $Y_{10}$ \\
$X_{11}$ & 0 & 0 & 0 & 0 & 0 & 0 & 0 & 0 & $Y_{11}$ \\
$X_{12}$ & 0 & 0 & 0 & 0 & 0 & 0 & 0 & 0 & $Y_{12}$ \\
$X_{13}$ & 0 & 0 & 0 & 0 & 0 & 0.011031 & 0 & 0.005203 & $Y_{13}$ \\
$X_{14}$ & 0 & 0 & 0 & 0 & 0.001746 & 0.001746 & 0.001746 & 0.001746 & $Y_{14}$ \\
$X_{15}$ & $g(E)$ & 0 & $\tilde{g}(E)$ & 0 & 0 & 0.105536 & 0 & 0.054944 & $Y_{15}$ \\
$X_{16}$ & 0 & 0 & 0 & 0 & 0.024467 & 0.024467 & 0.024467 & 0.024467 & $Y_{16}$ \\
$X_{17}$ & $E^{4}$ & $E^{4}$ & $E^{4}$ & 0 & 0.190351 & 0.190351 & 0.190351 & 0.190351 & $Y_{17}$ \\
$X_{18}$ & 0 & 0 & 0 & 0 & 0.018793 & 0.018793 & 0.018793 & 0.018793 & $Y_{18}$ \\
$X_{19}$ & 0 & 0 & 0 & $3^{-4 / 3}$ & 0.023467 & 0.023467 & 0.023467 & 0.023467 & $Y_{19}$ \\
\hline \hline
\end{tabular}


TABLE II. Eigenvalues and critical exponents of the recursion system linearized around the relevant fixed points given in Table I.

\begin{tabular}{lcccccccc}
\hline \hline & I & II & III & IV & V & VI & VII & VIII \\
& SAW & CP & SAW-CP & T & BP & SAW-BP & BP-CP & S-BP-CP \\
\hline$\lambda_{1}$ & 2.79645 & 4.0 & 3.70370 & 4.0 & 3.06817 & 3.06817 & 4.0 & 3.70370 \\
$\lambda_{2}$ & & & 2.22222 & $1.0^{\mathrm{a}}$ & & 2.79652 & 3.06817 & 3.06817 \\
$\lambda_{3}$ & & & & & & & & 2.22222 \\
$\nu$ & 0.67404 & 0.5 & 0.52939 & 0.5 & 0.61828 & 0.61828 & 0.5 & 0.52939 \\
$\phi$ & & & 0.60986 & & & 0.91731 & 0.80869 & 0.85622 \\
$\alpha$ & & & 0.36028 & & & 0.90985 & 0.76343 & 0.83208 \\
\hline \hline
\end{tabular}

${ }^{a}$ This marginal eigenvalue corresponds to an unstable manifold of the nonlinear equations. The corresponding direction is marginally repulsive.

with $n_{3}=n_{4}=1, n_{7}=n_{15}=n_{16}=-2, n_{8}=-3$, $n_{9}=n_{10}=-1, n_{11}=n_{19}=2, n_{17}=-4$, and the remaining $n_{j}$ equal to zero, in our case. Dropping the sixth component, we can then rewrite the remaining recursion equations as a nonlinear transformation

$$
\vec{Y}^{\prime}=\vec{T}(\vec{Y})
$$

for the 18-component vector $\vec{Y}$. If this vector goes to a finite fixed point $\vec{Y}^{*}$, then also the ratio $X_{6}^{\prime} / X_{6}$ reaches a finite fixed point value.

We have collected in Table I the fixed points of all three types that are relevant for our further discussion, and we will refer to them by the corresponding roman number. In addition, we report in Table II the eigenvalues associated with the linearized transformation around the fixed points of Table I, and the corresponding critical exponents.

In order to discover which, among these fixed points, play a role for our problem, it is necessary to iterate the 19 recursions numerically, starting from initial values given in terms of the bare physical parameters. The initial values of the zeroth-order tetrahedra partial generating functions are

$$
\begin{aligned}
X_{1} & =x \\
X_{2} & =x^{2} v^{4} \\
X_{3} & =x^{2} u^{1 / 2} v \\
X_{4} & =x^{3} u^{3 / 2} \\
X_{11} & =x^{3} u v^{3} \\
X_{19} & =x^{4} u^{2} v^{2}
\end{aligned}
$$

and $X_{j}=0$ for the other $j$ 's.

We now describe the behavior of the vector $\vec{X}$ (or $\vec{Y}$ ) under the renormalization flow, starting from these values.

(1) For $u \leq 1$ the monomer-monomer interaction $v$ is dominant and the polymer shows a $\Theta$ transition from a linear to a compact regime of the type studied in Ref. [17].

In the range of $v<v_{\Theta}$ the system of equations flows towards the SAW fixed point I. Linearizing the recursion equations about fixed point $\mathrm{I}$, we find one relevant eigenvalue $\lambda_{\mathrm{SAW}} \equiv 2^{y_{\mathrm{SAW}}}=2.79645$ which gives a critical exponent $\nu=\ln 2 / \ln \lambda_{\mathrm{SAW}}=0.67404$, the same $\nu$ exponent obtained for other models of a SAW on 3D Sierpinski gaskets $[17,21]$. This result is rather natural because on this gasket, as in the case of Euclidean lattices, for a one-tolerant trail silhouette model with not strong enough attractive interactions, only excluded volume effects can affect the thermodynamical behavior of the system, giving rise to the physics of swollen linear polymers.

By fixing precisely the monomer-monomer interaction at the value $v=v_{\Theta}(u)$, the basin of attraction of fixed point III is reached. The linearized renormalization transformation around this fixed point has two relevant eigenvalues: $\lambda_{1}=100 / 27$ and $\lambda_{2}=20 / 9$. This is the tricritical point corresponding to the usual $\Theta$ chain behavior, with a radius of gyration exponent $\nu_{\theta}=\ln 2 / \ln \lambda_{1}=0.52939$ [17]. The crossover exponent is $\phi=\ln \lambda_{2} / \ln \lambda_{1}=0.60986$ and the relative thermal exponent is $\alpha=2-1 / \phi=0.36028$. To get an idea of the location of this $\Theta$ line, notice that for $u=1$ we have $v_{\Theta} \simeq 1.4$.

For $v>v_{\Theta}$ the RG recursion equations flow towards the fixed point II. We note that for this fixed point the only surviving configuration in the thermodynamic limit is the one described with $X_{2}$; in this configuration all sites are occupied (see Fig. 1), suggesting the possibility of a compact phase with a finite density of monomers per site. In fact the linearization around point II gives only one relevant eigenvalue $\lambda_{c}=4$ (the subscript $c$ means "compact") with a corresponding critical exponent $\nu_{c}=$ $\frac{\ln 2}{\ln 4}=1 / \bar{d}$, where $\bar{d}$ is the fractal dimension of the $3 \mathrm{D}$ Sierpinski gasket, confirming that indeed the polymer is in a compact phase.

(2) For fixed $u \gg 1$ and $v$ varying, the twice visited site interaction prevails over the monomer-monomer attraction and we observe a new physical behavior, analogous to that found for the previously mentioned 2D model [13]. The strength of this twice visited site interaction opens the possibility for the polymer to assume a branched structure. Between the regions corresponding to a swollen structure (SAW) and to a collapsed structure (CP), which are dominated by the fixed points I and II, there is a region in the phase space that is dominated by fixed point $\mathrm{V}$ which describes the branched polymer (BP). The transitions between these three regions are 
controlled by the tricritical points VI and VII, both with two relevant eigenvalues. All the fixed points related to this branched structure are of the second type described above, i.e., the parameters $X_{j}$ have to be rescaled to parameters $Y_{j}$ according to (1.9).

It should be pointed out that the numerical determination of the critical parameter $x=x_{c}(u, v)$ is rather difficult in the branched polymer region, especially close to the CP phase. This is due to the fact that the phase trajectory of the variables $\vec{Y}$ under renormalization comes in the neighborhood of the fixed point $T$, also reported in Table I. However, this fixed point can never be reached from physical values for $u$ and $v$. In order to arrive in the neighborhood of the branched polymer fixed point $\mathrm{V}$, one would have to start from the exact $x_{c}$ with a precision of several hundred digits. In order to verify the fact that the whole region between the SAW phase and the collapsed phase is dominated by the same fixed point $\mathrm{V}$, we have therefore followed a different strategy. We worked with a precision of 40 digits (in MATHEMATICA) and determined for given values of $u$ and $v$ two values $x_{c 1}$ and $x_{c 2}$, almost equal to each other but leading, respectively, to the trivial fixed points $\vec{Y}=\overrightarrow{0}$ and $\infty$. The true $x_{c}(u, v)$ must then lie between these two. Along the trajectories starting from these $x_{c j}$, we determined two points $\vec{Y}_{1}$ and $\vec{Y}_{2}$ in the parameter space, both in the neighborhood of the fixed point $T$, and sufficiently far apart from each other to allow reasonable interpolations with the working precision of the computer. We then generated new iterations starting from intermediate values $\alpha \vec{Y}_{1}+(1-\alpha) \vec{Y}_{2}$, adjusting the value of $\alpha$ in the search for the final fixed point. Since this could still not be achieved by a 40-digit precision on $\alpha$, the same procedure had to be repeated several times, until it became finally clear that the iteration really reached the fixed point $\mathrm{V}$.

A transition between $\mathrm{SAW}$ and $\mathrm{BP}$ regimes has previously been observed, for example, in the study of twodimensional vesicle models with an associated osmotic pressure difference $\Delta p$ between interior and exterior, either on Euclidean [22] or on fractal [23] lattices. More specifically, in an exact study of a vesicle model on a fractal lattice [23] it has been shown that the perimeter of the vesicle in the flaccid regime, corresponding to $\Delta p=0$, behaves as a self-avoiding ring (with a $\nu$ exponent characteristic of SAW behavior). As soon as the vesicle is deflated $(\Delta p<0)$, it falls in the universality class of the $\mathrm{BP}$, and there is no multicritical fixed point separating the two different regimes; the role of the multicritical point is in this case played by the SAW fixed point, corresponding to $\Delta p=0$. This behavior reproduces exactly the one found for previously studied models on Euclidean lattices [22].

The deep difference between the situations described in the above-mentioned works and the model treated in this section consists in the presence, in the latter case, of an extra multicritical point, with its own exponents, separating SAW from BP regimes; we stress that such a point has also been found by some of the present authors in a study of a similar two-dimensional model [13].
In the $\mathrm{BP}$ regime only one relevant eigenvalue $\lambda_{\mathrm{BP}}=$ 0.306817 is present, giving the exponent $\nu_{\mathrm{BP}}=0.61828$. This exponent is slightly larger than the one found in a previous study of a collapsing BP in the 3D Sierpinski gasket involving only 11 partial generating functions [18]. The small discrepancy is not due to different levels of accuracy. In fact, one has to conclude that there is a slight nonuniversality in the way the two models on the $3 \mathrm{D}$ gasket are reproducing the properties of the $\mathrm{BP}$ scaling regime. A priori, similar or even more conspicuous discrepancies could have been found for the other regimes. Altogether it is certainly a point in favor of hierarchical lattices that they tend to respect, either strictly or to a high degree of precision, universalities that we would expect to hold on Euclidean lattices.

At the multicritical fixed point separating the branched from the collapsed phase, we have again two relevant eigenvalues. The corresponding exponent $\nu=0.5$ is in good agreement with that given in Ref. [18] for the $\Theta$ point of the collapsing BP, but the crossover exponent is quite different, resulting here in a more singular free energy, with a positive $\alpha$ exponent. This difference should be attributed to the richer structure of the allowed polymer configurations: the fact that sites may be visited twice allows for a different transition to the branched polymer phase. It is not clear to us whether the nonuniversality of the crossover exponent with respect to that of Ref. [18] is only a consequence of the hierarchical lattice, or follows from more general reasons.

(3) Where the strength of the two competing parameters $u$ and $v$ is comparable $(u \sim 1.8, v \sim 1.6)$ the three multicritical lines converge into a supermulticritical point. Due to its highly repulsive nature, it has not been possible to find the precise location of the point in the $(u, v, x)$ space that is flowing into this fixed point, but the root finding algorithm of MATHEMATICA delivered the values reproduced in Tables I and II for the fixed point VIII with three relevant eigenvalues.

We present a qualitative phase diagram of our model in Fig. 3.

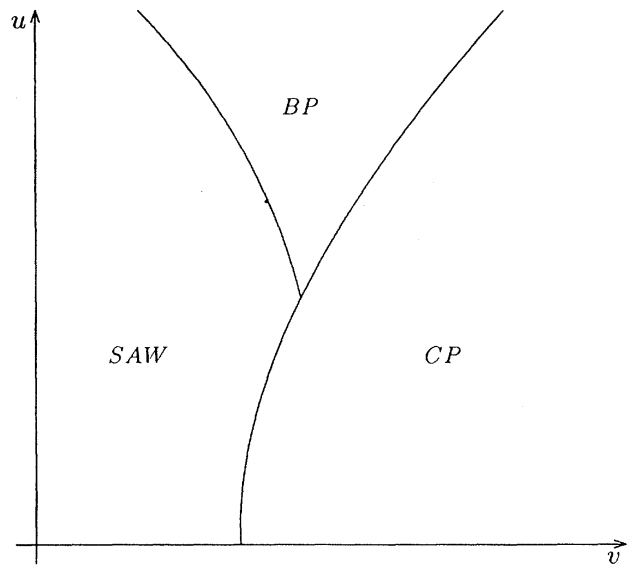

FIG. 3. Schematic representation of the phase diagram of the model in Sec. I. 


\section{Discussion}

We have presented exact results for a model of linear interacting polymers embedded in a 3D Sierpinski gasket. Varying the two parameters $u$ and $v$, which tune the effects of competing interactions, we obtain a rich phase diagram characterized by different swollen and compact phases of the polymer and corresponding multicritical transitions between them. In particular, we observe a transition from a swollen linear phase to a swollen branched phase, the two phases being separated by a tricritical point with its own exponents. This transition is followed by a next multicritical transition into the completely collapsed phase. Moreover, we have been able to show the presence of a higher order multicritical point, that separates the regions attracted by the three tricritical points between pairs of phases. We do not believe that these features are solely due to the nature of the gasket; in fact, as we will see in the next sections, a transition from SAW to BP regimes, followed by a collapse into a compact phase, can also be observed on Euclidean lattices.

Another result that we could verify exactly for our hierarchical model is the absence of a transition separating the CP into different regimes. This occurs in spite of the fact that the RG recursions allow for two distinct fixed points (II and IV) with $\nu=1 / \bar{d}=1 / 2$, describing compact regimes. The presence of more than one such fixed point is a clear prerequisite for the realization of a scenario with two distinct CP phases. Fixed point IV, however, has two unstable directions and can therefore never be reached from physical initial conditions. It can be considered as a multicritical point, separating our physical part of the phase space from the unphysical part with $X_{6}<0$. Since one of the unstable directions is only marginally so, it cannot be excluded that some small variations in the physical model might eventually stabilize this marginality and turn it into a second physically accessible compact fixed point. Such new models would then exhibit two distinct compact phases with a sharp transition in between (controlled by a new multicritical point).

\section{RSRG ON A 2D EUCLIDEAN LATTICE}

\section{A. Introduction}

The RG treatment of the previous section, though applied to a hierarchical lattice, has the important virtue of being exact. While interesting in its own right, this kind of approach is also believed to give a qualitative picture of the physics on more realistic lattices. As a matter of fact, the use of a well defined hierarchical structure is a clean way for avoiding the proliferation problems inherent in any RG approach on regular lattices. Thus, an exact approach to a problem on a hierarchical lattice has certainly also a value as an approximate treatment for the same problem on regular lattices, where arbitrary truncations would be unavoidable.

With such a perspective, taking into account the considerable mathematical complexity of the calculations in the previous section, it is natural to ask whether the physics obtained there can also be described within relatively more simple approximate schemes, which are not intended to be exact on some hierarchical lattice. Working within such approximate RG schemes directly should offer a more immediate and visualizable description of the phenomena, in terms of flows in relatively low dimensional parameter spaces.

In order to set up an approximate RG transformation with such features, one has to choose a suitable model and work on it with the techniques of the real space $R G$ (RSRG) approach to polymer statistics. RSRG methods have been used to study different problems of polymer statistics during the last few years (for an early review see Ref. [24]).

Several applications of RSRG to polymers have been limited to the noninteracting SAW [25-28]. The first attempt to study linear and branched polymers embedded in a two-dimensional lattice and the crossover between them is due to Family [29]. He developed a twoparameter RSRG: besides the usual step fugacity $x$, also the concentration $b$ of polyfunctional units was renormalized. Two fixed points in the $(x, b)$ plane were found: the SAW fixed point with two relevant eigenvalues and the BP fixed point with one relevant eigenvalue. The SAW fixed point turned out to be unstable; in fact, as soon as the parameter $b$ was switched on, the renormalization flow moved towards the BP fixed point. In this scheme there is no multicritical point separating the two phases; this role is played by the SAW fixed point, similarly to the situation encountered in Ref. [23] for vesicle models.

RSRG techniques applied to the study of the usual $\Theta$ point for two-dimensional SAW's appeared for the first time in Ref. [30]. These authors studied a two-tolerant trail [16] with an energy favoring overlaps of two different steps on the same lattice bond, and gave for the $\nu$ exponent on the square lattice the values $\nu=0.73$ in the swollen phase, $\nu_{\theta}=0.66$ at the $\Theta$ point, and $\nu=0.62$ in the collapsed phase. However, it has been pointed out in Ref. [31] that, because of the special kind of interaction considered, this result is more likely to describe a collapse into branched polymers, whose $\nu$ exponent in $d=2$ is about $\nu \simeq 0.64$ [32]. Note that the $\nu_{\theta}$ exponent reported above agrees very well with that obtained for the SAW-BP multicritical point in Ref. [13] and also in our model described in the next section. The physics of the $\Theta$-point transition for the SAW within a RSRG approach was also treated in Ref. [33]. In both two and three dimensions the results of this study turned out to be qualitatively consistent with other numerical predictions and theoretical conjectures. In particular, the collapsed phase was correctly described in this approach.

We also want to mention the work of $\mathrm{Wu}$ and Bradley [34], which investigates $\Theta$ points by means of a threeparameter RSRG in the attempt to elucidate the universality classes of SAW's and self-avoiding trails (SAT's).

\section{B. The model}

We consider walks on the square lattice. The bonds of the lattice can be visited once (in order to model linear 
polymers), or twice. Favoring twice visited edges with respect to singly visited ones allows a possible mechanism of transition from swollen linear to swollen BP behavior. Indeed, dominant configurations should look like tree structures, with mutually excluding branches made of twice visited bonds. We again assign a fugacity $v$ to all monomer-monomer interactions. In this model, however, this means that an attractive energy is attributed to each pair of nearest neighbor bonds nonconsecutively visited either by single or by double steps. Furthermore, we introduce two different step fugacities: $x$ is the once visited step fugacity and $p$ is the twice visited step fugacity. The grand canonical partition function can then be written as

$$
\mathcal{Z}(x, p, \mu)=\sum_{N_{1}, N_{2}, l=0}^{\infty} C\left(N_{1}, N_{2}, l\right) x^{N_{1}} p^{N_{2}} v^{l}
$$

where $N_{1}, N_{2}$, and $l$ are, respectively, the number of once or twice visited bonds and the number of nearest neighbor interactions. $C\left(N_{1}, N_{2}, l\right)$ is the number of different configurations of the polymer with fixed values of $N_{1}$, $N_{2}$, and $l$. Note that for $N_{2}=0$ we have self-attracting linear polymers and vice versa.

\section{Method and results}

We work on a square lattice and partition it into cells that cover the lattice while maintaining its original symmetry. Often in the RSRG approach to critical phenomena of polymer systems an $M \times M$ cell is renormalized into an $L \times L$ cell, with $M$ and $L$ integers such that $1 \leq L<M$. Under this transformation the correlation length is rescaled by a factor $L / M$.

The fugacities $x^{\prime}, p^{\prime}$, and $\mu^{\prime}$ in the rescaled system are given in terms of the old ones by recursion equations

$x^{\prime}=x^{\prime}(x, p, v) ; \quad p^{\prime}=p^{\prime}(x, p, v) ; \quad v^{\prime}=v^{\prime}(x, p, v)$.

Critical exponents characterizing different regimes of the system are obtained in terms of the eigenvalues of the renormalization transformation, linearized around relevant fixed points. In particular the $\nu$ exponent controlling the divergence of the correlation length at criticality is given by $\nu=\ln b / \ln \lambda_{+}$where $b=M / L$ is the rescaling factor and $\lambda_{+}$is the largest eigenvalue of the renormalization transformation. We have obtained the renormalized $x^{\prime}$ and $p^{\prime}$ by considering $2 \times 2$ cells rescaled onto $1 \times 1$ cells, so that the rescaling factor is $b=2$. A basic step in the renormalization procedure consists in choosing which polymer configurations within a cell should contribute to a renormalized bond. We map a walk within a cell into a rescaled bond if the walk traverses (i.e., spans or gets across) the cell; in our case this means that it has to enter the cell at site 1 and to come out at site 2 or 3 [see Fig. 4(a)]. We restrict the entrance to the cell to a corner (e.g., the lower left corner), following the so-called corner rule [24]. We want to stress that other definitions
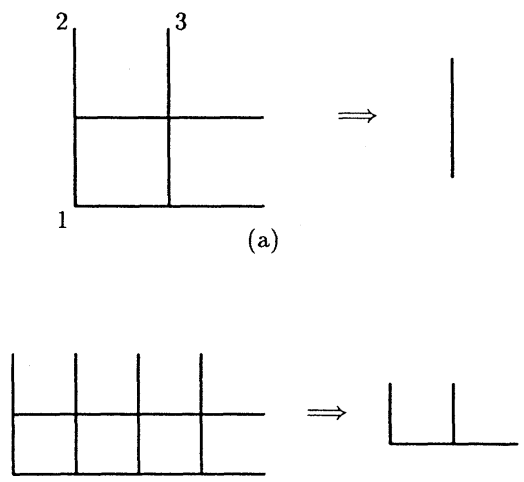

(b)

FIG. 4. Cell renormalization chosen for the model in Sec. II: (a) cell renormalization for the fugacities $x$ and $p$; (b) cell renormalization for the fugacity $v$.

of "getting across" may be used, and it is expected that all acceptable rules would converge to the same results in the large cell limit.

We now describe our scheme for renormalizing $x$ and $p$. We allow one or two chains to come in through the origin 1. If two chains share the same bond, this bond contributes with a factor $p$, otherwise it contributes with a factor $x$. Moreover, a walk can fold onto itself and retrace twice the same bond; also in this case there is a $p$ contribution. This folding is allowed also if the chain is in site 2 or 3 , by which we mean that the chain in 2 or 3 is not obliged to come out of the cell (see Fig. 5).
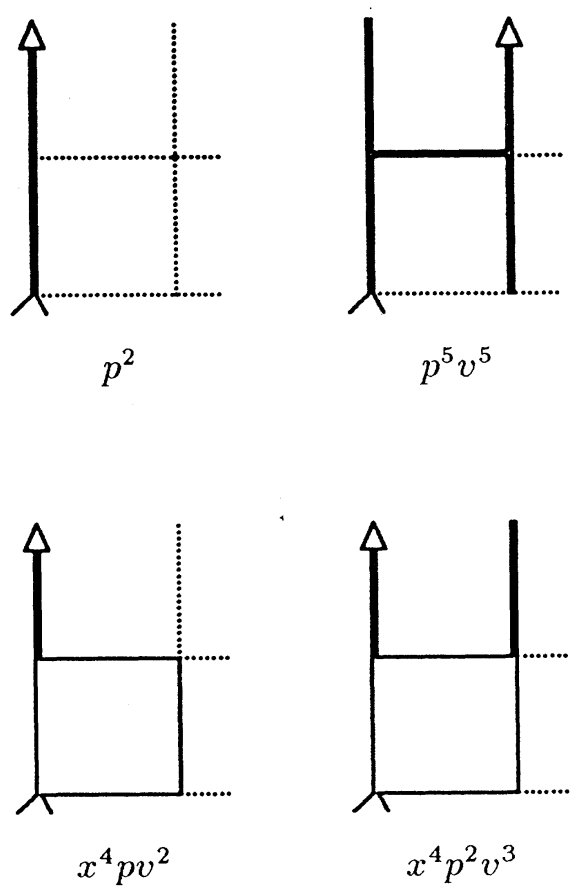

FIG. 5. Examples of configurations renormalizing into $p^{\prime}$. 


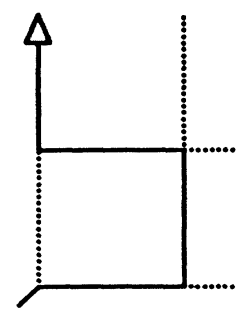

$x^{4} v$

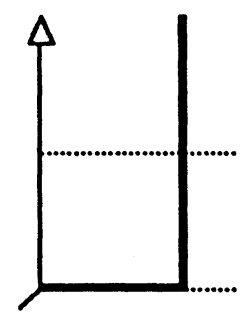

$x^{2} p^{3} v^{2}$

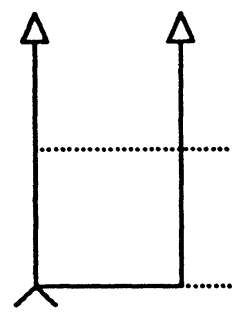

$x^{5} v^{2}$

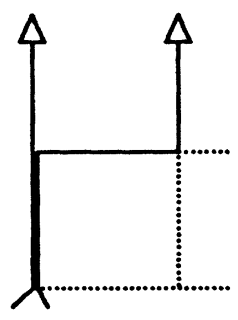

$x^{3} p v$
FIG. 6. Examples of configurations renormalizing into $x^{\prime}$.
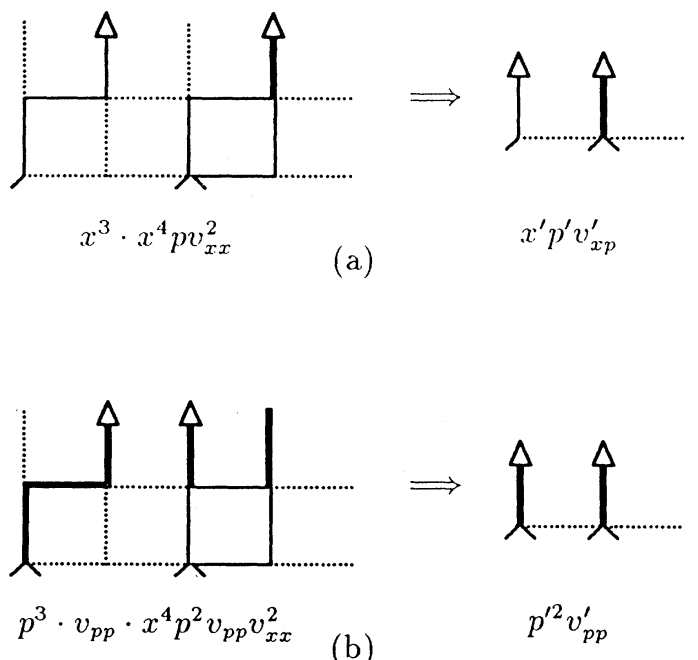

FIG. 7. Examples of proliferation in the renormalization of $v$. The factorization of the contributions is given as an indication of their origin.
Configurations in which one end is occupied by a $p$ contribution and the other end is empty or in which both ends are spanned by a double line (see Fig. 5 for some examples) will contribute in the renormalization transformation to the $p^{\prime}$ fugacity; all remaining configurations will contribute to the $x^{\prime}$ fugacity. Note that among configurations renormalizing into $x^{\prime}$ we consider also, rather arbitrarily, cells with one end occupied by an $x$ step and the other end by a $p$ step (see Fig. 6).

For the renormalization of the parameter $v$, we follow a procedure previously used in Ref. [33], consisting in the mapping of a $4 \times 2$ cell into a $2 \times 1$ cell [see Fig. 4 (b)]. The renormalized interaction $v^{\prime}$ is obtained by considering all configurations that simultaneously span two adjacent $2 \times 2$ cells. This in principle gives rise to different fugacities $v_{x x}^{\prime}, v_{x p}^{\prime}$, and $v_{p p}^{\prime}$ according to the type of crossed cells (Fig. 7). Since we would like to avoid such a proliferation of parameters, we introduce an extra approximation by defining an appropriate averaging

$$
v^{\prime} \equiv \frac{x^{2} v_{x x}^{\prime}+2 x^{\prime} p^{\prime} v_{x p}^{\prime}+p^{\prime 2} v_{p p}^{\prime}}{x^{\prime 2}+2 x^{\prime} p^{\prime}+p^{\prime 2}}
$$

Along these lines, using the computer to enumerate all possible configurations, we obtain a set of three recursion equations for three parameters. The full recursions are reproduced in the Appendix.

The analysis of the system of equations and of the renormalization flow gives a set of relevant fixed points reported in Table III along with their corresponding eigenvalues and critical exponents $\nu$. Apart from the fixed point at $v=\infty$, corresponding to the collapsed phase, we find three fixed points in the $v=1$ plane: a SAW point ( $A$ in Fig. 8) with $p=0$, a BP point $(B)$ with $x=0$, and a multicritical point $(C)$ in between, governing the crossover between the two previously mentioned points. While $A$ and $B$ are characterized by only one relevant eigenvalue, $C$ is a multicritical point having two eigenvalues greater than 1; it controls the SAW-BP transition. For attractive interactions $(v>1)$ we have the usual $\Theta$ fixed point for SAW's $(D)$ characterized by two relevant eigenvalues and the analogous $\Theta$ fixed point for BP's $(E)$ separating the swollen branched phase from the compact one. Finally we have a highly repulsive multicritical point $(F)$ with three relevant eigenvalues governing the entire flow diagram.

The RG flow in the parameters $x, p$, and $\ln v$ is visualized in Fig. 8. We verified that the fixed point structure

TABLE III. Eigenvalues and critical exponents for the model in Sec. II.

\begin{tabular}{|c|c|c|c|c|c|c|c|}
\hline & $\begin{array}{c}A \\
(\mathrm{SAW})\end{array}$ & $\begin{array}{c}B \\
(\mathrm{BP})\end{array}$ & (CP) & $\begin{array}{c}C \\
\text { (SAW-BP) }\end{array}$ & $\begin{array}{c}D \\
\text { (SAW-CP) }\end{array}$ & $\begin{array}{c}E \\
(\mathrm{BP}-\mathrm{CP})\end{array}$ & $\begin{array}{c}F \\
(\mathrm{~S}-\mathrm{BP}-\mathrm{CP})\end{array}$ \\
\hline$\overline{x^{*}}$ & 0.45339 & 0.0 & 0.0 & 0.28338 & 0.37906 & 0.0 & 0.22840 \\
\hline$p^{*}$ & 0.0 & 0.29780 & 0.0 & 0.29546 & 0.0 & 0.23942 & 0.24538 \\
\hline$v^{*}$ & 1.0 & 1.0 & $\infty$ & 1.0 & 2.91142 & 2.37160 & 2.07446 \\
\hline$\lambda_{1}$ & 2.72430 & 3.13766 & & 3.17285 & 3.28742 & 3.66305 & 3.78401 \\
\hline$\lambda_{2}$ & & & & 2.40046 & 1.55590 & 1.43933 & 2.42034 \\
\hline$\lambda_{3}$ & & & & & & & 1.84678 \\
\hline$\nu$ & 0.69162 & 0.60617 & & 0.60032 & 0.58243 & 0.53389 & 0.52086 \\
\hline
\end{tabular}




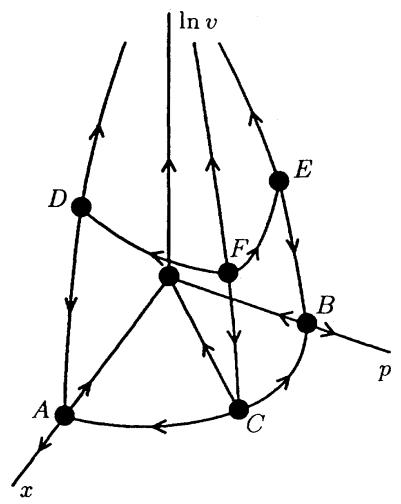

FIG. 8. Schematic representation of the flow diagram of the model studied in Sec. II.

is consistent with a phase diagram qualitatively similar to that already reported in Fig. 3, with the interactions $v$ and $p$ here playing roles corresponding to $v$ and $u$ there, respectively.

So, also for the model treated by RSRG in this section, the competition between the compactifying interaction, $v$, and $p$, which controls the formation of double bonds, gives rise to the transitions and regimes we wanted to identify. It is somehow remarkable that the complicated fixed point structure sketched in Fig. 8 could be associated with RG recursions certainly based on drastic approximations, albeit full of different contributions.

\section{EXACT ENUMERATION FOR A MODEL ON 2D EUCLIDEAN LATTICE: SERIES EXPANSIONS FOR THE FULL PHASE DIAGRAM}

\section{A. The model}

The approaches in both previous sections have mostly a qualitative value and give results which are highly suggestive of the possibility that a polymer system with suitable interactions could display the rich phase diagram already discussed, encompassing SAW's, BP's, and CP's. In an attempt towards a more quantitative characterization of the various regimes and of the transition between them, we also decided to apply exact enumerations analysis to a system on the square lattice with competing interactions. Although we also studied series expansions for other models (e.g., two-tolerant trails with bond overlap fugacity and nearest neighbor attractive interaction), the most satisfactory numerical results were obtained for the one described below.

Consider self-avoiding walks on the square lattice, in which, as in Sec. I, a fugacity $x$ is given for each step and a fugacity $v$ for every pair of nonconsecutive nearest neighbor sites on the walk. These two fugacities lead to the usual $\Theta$ transition between SAW and CP. In order to enhance the formation of thin branchlike structures, we have in addition given a fugacity $y$ to every step of the walk that separates two squares whose four corners

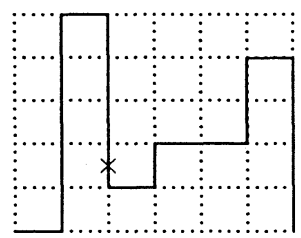

(a)

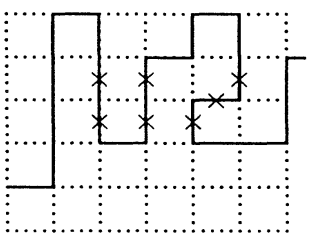

(b)
FIG. 9. Examples of walks for the model in Sec. III: contributions are $x^{22} v^{7} y$ from (a) and $x^{23} v^{12} y^{7}$ from (b). Crosses indicate the origins of the $y$ factors.

all belong to the walk. We have illustrated this idea for two walks in Fig. 9, where we indicated with the symbol $\times$ each step that gives rise to an extra factor $y$. A simple branch formed by parallel steps will not generate $y$ fugacity factors; once such a branch is formed, however, every attempt to align to it a new step of the walk, as in Fig. 9(a), will generate (apart from the standard fugacity $v$ ) also this fugacity $y$. For sufficiently large values of $v$ we then expect to find a transition from the collapsed phase to the branched phase if we decrease the value of $y$. Physically, this $y$ parameter represents a reduction in the monomer-monomer interaction due to saturation by other neighboring monomers. If we fix $y=1$, we should get back the previously studied linear polymer model $[35,36,4]$ where a $\Theta$ transition is expected at $v_{c} \approx 1.95[4]$.

\section{B. Method and results}

We have enumerated all possible SAW's up to 26 steps, and counted the number of $v$ and $y$ factors that should be associated with them. Furthermore, we have also calculated their radius of gyration. Previous experience has taught that the radius of gyration leads in general to a faster convergence for the asymptotic analysis of series expansions than the end-to-end distance. In this way we obtained the numbers $C(N, i, j)$ and $R(N, i, j)$, representing, respectively, the total number of walks and the accumulated radius of gyration corresponding to $N$ steps, $i$ nearest neighbor interactions, and $j$ steps sandwiched between two fully visited squares. For each set of values $(v, y)$ we can calculate the average radius of gyration of an $N$-step walk by

$$
\langle R(v, y)\rangle_{N}=\frac{\sum_{i} \sum_{j} R(N, i, j) v^{i} y^{j}}{\sum_{i} \sum_{j} C(N, i, j) v^{i} y^{j}} .
$$

If this $\langle R\rangle_{N}$ behaves asymptotically as $N^{\nu}$, we then expect that the generating function $F(x)$, defined by

$$
F(x) \equiv \sum_{N}\langle R(v, y)\rangle_{N} x^{N}
$$

should behave as 


$$
F(x) \approx A(1-x)^{-(1+\nu)}
$$

when $x \rightarrow 1$.

Since 26 is still a small number, we cannot expect to find in the $(v, y)$ plane sharp transitions between regions with constant $\nu$ values. Nevertheless, we can clearly distinguish the three expected phases through large regions in which $\nu$ remains approximately constant. For weak monomer-monomer interactions $(v \approx 1)$ we have the swollen SAW phase with $\nu=0.75$. For strong interactions (typically $v>3$ ) and $y \approx 1$, we have a collapsed phase (CP) with $\nu=0.5$; for the same $v$ values but low $y$ $(y \approx 0)$, we have the branched polymer (BP) phase with $\nu \approx 0.6$.

In order to determine more precisely the transition between the different regions of the phase diagram, one can use the scaling relation suggested by de Gennes [37] for finite chains in the neighborhood of the $\Theta$ point:

$$
\langle R(v)\rangle_{N} \propto N^{\nu} F\left(N^{\phi} \Delta v\right)
$$

with $\Delta v=v-v_{\Theta}$. A crossover form like (3.4) can of course be applied also to the other transitions that we expect to meet in our model. This allows a nice numerical determination of both $v_{\Theta}$ and $\nu$, since this equation implies that the curves

$$
f_{N, M}(v) \equiv \frac{\ln \left[\langle R(v)\rangle_{N} /\langle R(v)\rangle_{M}\right]}{\ln (N / M)}
$$

cross each other at $v=v_{\Theta}$, where they attain the multicritical value of $\nu[36,4]$. Using this criterion, we can determine both the $\nu$ between SAW and CP and that between SAW and BP phases. Due to the odd-even oscillations typical for the square lattice, we only use the functions $\bar{f}_{N} \equiv f_{N, N-2}$. In Fig. 10 we reproduce a typical example of such curves, exhibiting here two intersection points. We observe that in the SAW phase the functions $\bar{f}_{N}$ increase with increasing $N$ values, while they decrease with $N$ in the collapsed and BP phases; at the respective

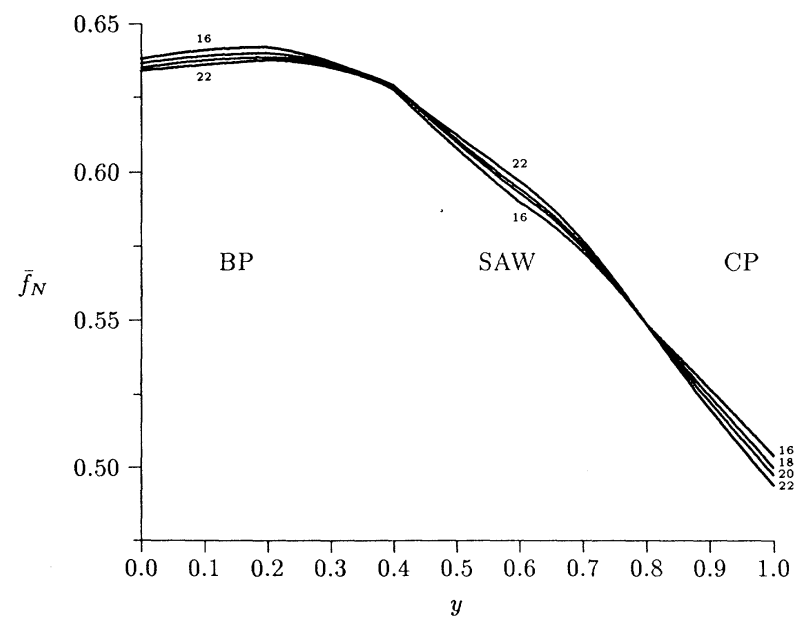

FIG. 10. Double intersections for functions $\bar{f}_{N}(y)$ at $v=2.7$; the numbers on the curves indicate the values of $N$.

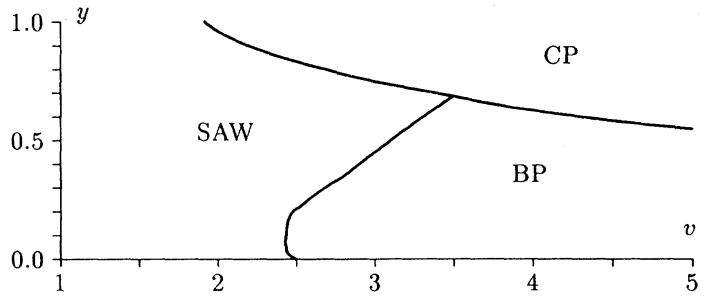

FIG. 11. Phase diagram for the series expansion of the model in Sec. III.

transitions they are stable, which leads to an intersection point. From these facts, however, we can hardly expect to find an intersection of the same lines at the transition line between CP and BP phases: we can at most expect a point of close encounter, since these functions decrease with increasing $N$ in both phases. One criterion which we applied for this last transition line would then be to choose the point where all these $f$ values have the smallest variance, or where there is an intersection point for the derivatives of these functions. We have also applied other, more sophisticated, methods of series analysis for determining these transition lines, and they are all consistent with each other within a few percent. The resulting phase diagram is shown in Fig. 11. Along these transition lines one can analyze the critical exponents $\nu$ and $\phi$, as defined in (3.4). Our best estimates from this analysis are given in Table IV.

The $\nu$ exponent for the SAW-BP transition appears compatible with that obtained in Ref. [13] for a different model displaying the same transition $(\nu=0.66 \pm 0.03)$. Also the $\nu$ exponent for the SAW-CP collapse is close to the value expected in 2D. Our estimate of $\phi$ for this same transition is far off the expected exact value $(3 / 7)^{2}$, as is the case in all series determinations attempted so far. Our value of $\nu$ for the collapse of BP into CP does not fall far from one of the two which are presently most accredited [11] for the BP $\Theta$ transitions in $2 \mathrm{D}(\nu=1 / 2$ and $\nu=8 / 15)$.

Taking into account the obvious limitations affecting our extrapolations of exact enumeration data, the reasonable consistency of our determinations with known or expected exponents at the various multicritical transitions gives strong confidence that the features of the phase diagram already encountered in the hierarchical model and in the RSRG approximate treatment apply also to the model in this section.

TABLE IV. Critical exponents at the multicritical transitions for the model in Sec. III.

\begin{tabular}{llll}
\hline \hline & SAW-CP & SAW-BP & BP-CP \\
\hline$\nu$ & 0.57 & 0.65 & 0.56 \\
$\phi$ & 0.8 & 0.56 & 1.2 \\
\hline \hline
\end{tabular}




\section{DISCUSSION}

In this paper we have given evidence, based on different models and approaches, that a linear polymer system can display swollen, swollen branched, and compact regimes under the effect of a competition between interactions inducing mere ramification and interactions producing complete collapse. The resulting phase diagram is intriguing because, besides simultaneously including the more familiar $\Theta$ transition lines for linear and branched polymers, it displays the additional type of SAW-BP transition already predicted in Ref. [13]. The above three transition lines join at a highly unstable multicritical point.

Both our results for the hierarchical model and those based on enumerations in $2 \mathrm{D}$ seem to suggest that the linear polymer $\Theta$ transition should normally belong to the universality class expected for standard $\Theta$-point models $[2,17]$. For the BP's $\Theta$ transition, it is more difficult to draw precise conclusions. On the other hand, already in relatively more conventional $2 \mathrm{D}$ models of lattice animals and BP, at least two distinct universality classes with rather close values of $\nu$ are expected [11].

For the SAW-BP transition, which is certainly the most unconventional line in our phase diagrams, we got strong evidence of scaling controlled by a peculiar multicritical fixed point, distinct from those associated with both swollen linear and branched regimes. In this respect we are thus facing a different type of multicritical point, which is not met, e.g., in models of $2 \mathrm{D}$ vesicles, where cross-overs from SAW to BP regimes are also observed $[38,39]$. A precise characterization of the scaling properties at this multicritical point remains an interesting challenge for both numerical and theoretical approaches, especially in $2 \mathrm{D}$, where our understanding of linear and branched polymer $\Theta$ transitions has made important progress in recent years [10-12]. Our enumeration result for $\nu$ at the 2D SAW-BP transition appears compatible with that obtained in Ref. [13] for a different model. Another interesting point in our phase diagram is that joining the three multicritical lines. We could obtain a precise location of this point and of its exponents only for the hierarchical model.

The phase diagram that we found in our models depends of course on the competitive effects determined by the chosen interactions. It would also be impossible to find a SAW-BP transition in most of the standard models of $\Theta$ transitions studied so far in the literature. Indeed, these models normally include only one attractive interaction. An exception is the system studied in Ref. [13], which was initially conceived for describing a linear polymer $\Theta$ collapse [30,31]. In this case, however, the absence of competition leads to only SAW and BP as possible phases [13].

To establish whether real polymer systems can present competition effects leading to phase diagrams of the type we prospected remains, of course, an open issue. It is perhaps worth recalling that in $3 \mathrm{D}$ the $\nu_{\theta}$ of linear polymers is $1 / 2$, while the $\nu$ of swollen branched polymers is also $1 / 2$ [40]. We are confident that, at the theoretical level, our findings open an interesting and still largely unexplored chapter.

\section{ACKNOWLEDGMENTS}

We would like to thank F. Seno for collaboration in the early stages of this project and T. Bolzonella for useful discussions and criticism. We are also grateful for the kind hospitality at the Department of Physics of the University of Bologna. This work was supported financially by the INFN of Italy and by the NATO under Grant No. SA.9-15-04(RV.910413).

\section{APPENDIX}

The recursion equations obtained in Sec. II are as follows:

$$
\begin{aligned}
x^{\prime}= & x^{2}+2 x^{3}+v x^{4}+v^{2} x^{5}+p\left(2 x^{2}+6 v x^{3}+2 v^{2} x^{4}+v^{3} x^{5}\right) \\
& +p^{2}\left(4 v x^{2}+8 v^{2} x^{3}+v^{3} x^{4}\right)+p^{3}\left(4 v^{2} x^{2}+2 v^{3} x^{3}\right)+p^{4} v^{3} x^{2} \\
p^{\prime}= & 2 p v^{2} x^{4}+p^{2}\left(1+2 v^{3} x^{4}\right)+4 p^{3}+10 p^{4} v+10 p^{5} v^{2}+2 p^{6} v^{2} \\
v_{x x}^{\prime}= & x^{4}+x^{5}\left(2+v+v^{2}\right)+x^{6}\left(3+2 v+v^{2}\right)+x^{7}\left(v+4 v^{2}+v^{4}\right)+x^{8}\left(2 v^{2}+2 v^{3}+v^{4}\right) \\
& +2 x^{9} v^{4}+x^{10} v^{6}+p\left[4 x^{4}+x^{5}\left(4+8 v+5 v^{2}+3 v^{3}\right)+x^{6}\left(16 v+12 v^{2}+3 v^{3}+v^{4}\right)\right. \\
& \left.+x^{7}\left(5 v^{2}+17 v^{3}+3 v^{4}+v^{5}\right)+x^{8}\left(4 v^{3}+10 v^{4}+6 v^{5}\right)+x^{9}\left(4 v^{5}+2 v^{6}\right)+2 x^{10} v^{7}\right] \\
& +p^{2}\left[x^{4}\left(4+5 v+3 v^{2}\right)+x^{5}\left(18 v+20 v^{2}+12 v^{3}+6 v^{4}\right)+x^{6}\left(34 v^{2}+39 v^{3}+12 v^{4}+v^{5}\right)\right. \\
& \left.+x^{7}\left(8 v^{3}+36 v^{4}+12 v^{5}\right)+x^{8}\left(2 v^{4}+16 v^{5}+16 v^{6}\right)+x^{9}\left(2 v^{6}+4 v^{7}\right)+x^{10} v^{8}\right] \\
& +p^{3}\left[x^{4}\left(10 v+2 v^{3}\right)(1+v)+x^{5}\left(33 v^{2}+38 v^{3}+28 v^{4}+3 v^{5}\right)+x^{6}\left(30 v^{3}+73 v^{4}+33 v^{5}\right)\right. \\
& \left.+x^{7}\left(4 v^{4}+37 v^{5}+25 v^{6}\right)+x^{8}\left(8 v^{6}+18 v^{7}\right)+2 x^{9} v^{8}\right] \\
& +p^{4}\left[x^{4}\left(12 v^{2}+17 v^{3}+4 v^{4}+v^{5}\right)+x^{5}\left(28 v^{3}+53 v^{4}+47 v^{5}\right)+x^{6}\left(9 v^{4}+68 v^{5}+49 v^{6}\right)\right. \\
& \left.+x^{7}\left(14 v^{6}+24 v^{7}\right)+7 x^{8} v^{8}\right]+p^{5}\left[x^{4}\left(6 v^{3}+20 v^{4}+10 v^{5}\right)+x^{5}\left(8 v^{4}+43 v^{5}+49 v^{6}\right)\right. \\
& \left.+x^{6}\left(22 v^{6}+38 v^{7}\right)+8 x^{7} v^{8}\right]+p^{6}\left[x^{4}\left(v^{4}+11 v^{5}+12 v^{6}\right)+x^{5}\left(12 v^{6}+28 v^{7}\right)+11 x^{6} v^{8}\right] \\
& +p^{7}\left[x^{4}\left(2 v^{6}+6 v^{7}\right)+6 x^{5} v^{8}\right]+p^{8} x^{4} v^{8}
\end{aligned}
$$




$$
\begin{aligned}
& v_{x p}^{\prime}=p\left[x^{6}\left(2 v^{2}+v^{3}+v^{4}\right)+x^{7}\left(3 v^{2}+4 v^{3}+v^{4}\right)+x^{8}\left(v^{3}+3 v^{4}\right)+2 x^{9}\left(v^{5}+v^{6}\right)\right] \\
& +p^{2}\left[2 x^{2}+x^{3}\left(2+v+v^{2}\right)+x^{4}\left(v+v^{2}\right)+x^{5}\left(v^{2}+v^{4}\right)+x^{6}\left(4 v^{2}+2 v^{3}+4 v^{4}+2 v^{6}\right)\right. \\
& \left.+x^{7}\left(5 v^{3}+16 v^{4}+9 v^{5}+2 v^{6}\right)+x^{8}\left(v^{4}+5 v^{5}+6 v^{6}\right)+x^{9}\left(2 v^{6}+2 v^{7}+4 v^{8}\right)\right] \\
& +p^{3}\left[x^{2}\left(10+v+v^{2}\right)+x^{3}\left(10+10 v+5 v^{2}+3 v^{3}\right)+x^{4}\left(3 v+7 v^{2}+v^{3}+v^{4}\right)\right. \\
& +x^{5}\left(3 v^{2}+3 v^{3}+3 v^{4}+v^{5}\right)+x^{6}\left(3 v^{3}+13 v^{4}+4 v^{5}+4 v^{6}\right)+x^{7}\left(4 v^{4}\right. \\
& \left.\left.+20 v^{5}+22 v^{6}+10 v^{7}\right)+x^{8}\left(2 v^{6}+6 v^{7}+4 v^{8}\right)+4 x^{9} v^{9}\right]+p^{4}\left[x^{2}\left(12+18 v+11 v^{2}+3 v^{3}\right)\right. \\
& +x^{3}\left(45 v+38 v^{2}+15 v^{3}+6 v^{4}\right)+x^{4}\left(12 v^{2}+22 v^{3}+3 v^{4}+v^{5}\right)+x^{5}\left(5 v^{3}+12 v^{4}+11 v^{5}\right) \\
& \left.+x^{6}\left(v^{4}+11 v^{5}+12 v^{6}+8 v^{7}\right)+x^{7}\left(6 v^{6}+18 v^{7}+20 v^{8}\right)+4 x^{8} v^{9}\right] \\
& +p^{5}\left[x^{2}\left(38 v+38 v^{2}+15 v^{3}+9 v^{4}\right)+x^{3}\left(80 v^{2}+103 v^{3}+44 v^{4}+3 v^{5}\right)\right. \\
& \left.+x^{4}\left(14 v^{3}+43 v^{4}+11 v^{5}\right)+x^{5}\left(2 v^{4}+16 v^{5}+22 v^{6}\right)+x^{6}\left(2 v^{6}+6 v^{7}+12 v^{8}\right)+12 x^{7} v^{9}\right] \\
& +p^{6}\left[x^{2}\left(55 v^{2}+66 v^{3}+34 v^{4}+3 v^{5}\right)+x^{3}\left(64 v^{3}+160 v^{4}+88 v^{5}\right)+x^{4}\left(5 v^{4}+37 v^{5}+22 v^{6}\right)\right. \\
& \left.+x^{5}\left(6 v^{6}+18 v^{7}\right)+4 x^{6} v^{9}\right]+p^{7}\left[x^{2}\left(34 v^{3}+85 v^{4}+57 v^{5}\right)+x^{3}\left(16 v^{4}+124 v^{5}+104 v^{6}\right)\right. \\
& \left.+x^{4}\left(10 v^{6}+18 v^{7}\right)+4 x^{5} v^{8}\right]+p^{8}\left[x^{2}\left(7 v^{4}+51 v^{5}+58 v^{6}\right)+x^{3}\left(30 v^{6}+62 v^{7}\right)\right. \\
& \left.+4 x^{4} v^{8}\right]+p^{9}\left[x^{2}\left(10 v^{6}+26 v^{7}\right)+12 x^{3} v^{8}\right]+4 p^{10} x^{2} v^{8} \\
& v_{p p}^{\prime}=p^{2} x^{8}\left(3 v^{5}+v^{6}\right)+p^{3}\left[x^{4}\left(2 v^{2}+v^{3}+v^{4}\right)+4 x^{8}\left(v^{7}+v^{8}\right)\right] \\
& +p^{4}\left[1+x^{4}\left(7 v^{2}+6 v^{3}+5 v^{4}+2 v^{6}\right)+4 x^{8} v^{10}\right]+p^{5}\left[6+v+v^{2}+x^{4}\left(8 v^{3}+30 v^{4}\right.\right. \\
& \left.\left.+12 v^{5}+6 v^{6}\right)\right]+p^{6}\left[11+14 v+8 v^{2}+3 v^{3}+x^{4}\left(4 v^{4}+26 v^{5}+34 v^{6}+16 v^{7}\right)\right] \\
& +p^{7}\left[41 v+38 v^{2}+14 v^{3}+7 v^{4}+x^{4}\left(4 v^{6}+16 v^{7}+28 v^{8}\right)\right]+p^{8}\left(64 v^{2}+82 v^{3}+36 v^{4}\right. \\
& \left.+2 v^{5}+8 x^{4} v^{9}\right)+p^{9}\left(42 v^{3}+112 v^{4}+62 v^{5}\right)+p^{10}\left(8 v^{4}+67 v^{5}+65 v^{6}\right) \\
& +p^{11}\left(12 v^{6}+28 v^{7}\right)+4 p^{12} v^{8} \text {. }
\end{aligned}
$$

[1] A. Coniglio, N. Jan, I. Majid, and H.E. Stanley, Phys. Rev. B 35, 3617 (1987).

[2] B. Duplantier and H. Saleur, Phys. Rev. Lett. 59, 539 (1987).

[3] V. Privman, J. Phys. A 19, 3287 (1987).

[4] F. Seno and A.L. Stella, J. Phys. (Paris) 49, 739 (1988).

[5] I.S. Chang, Y. Shapir, and H. Meirovitch, J. Phys. A 23, L537 (1990).

[6] C. Vanderzande, A.L. Stella, and F. Seno, Phys. Rev. Lett. 67, 2757 (1991).

[7] D.P. Foster, E. Orlandini, and M.C. Tesi, J. Phys. A 25, 1211 (1992).

[8] D.S. Gaunt, Physica A 177, 146 (1991).

[9] S. Flesia, D.S. Gaunt, C.E. Soteros, and S.G. Whittington, J. Phys. A 25, L1169 (1992).

[10] C. Vanderzande, Phys. Rev. Lett. 70, 3595 (1993).

[11] F. Seno and C. Vanderzande, J. Phys. A 27, 5813 (1994).

[12] A.L. Stella, Phys. Rev. E 50, 3259 (1994).

[13] E. Orlandini, F. Seno, A.L. Stella, and M.C. Tesi, Phys. Rev. Lett. 68, 488 (1992); erratum (unpublished).

[14] R.M. Bradley, Phys. Rev. E 48, R4195 (1993).

[15] Y. Gefen, B.B. Mandelbrot, and A. Aharony, Phys. Rev. Lett. 45, 855 (1980).

[16] A. Malakis, J. Phys. A 8, 1885 (1975); 9, 1283 (1976).

[17] D. Dhar and J. Vannimenus, J. Phys. A 20, 199 (1987).

[18] M. Knezevic and J. Vannimenus, Phys. Rev. B 35, 4988 (1987).

[19] D. Dhar, J. Math. Phys. 19, 5 (1978).

[20] A request can be addressed to the electronic address raf.dekeyser@fys.kuleuven.ac.be

[21] E. Bouchaud and J. Vannimenus, J. Phys. (Paris) 50, 2931 (1989).
[22] S. Leibler, R.R.P. Singh, and M.E. Fisher, Phys. Rev. Lett. 59, 1989 (1987); M.E. Fisher, Physica D 38, 112 (1989).

[23] M. Di Stasio, F. Seno, and A.L. Stella, J. Phys. A 25, 3891 (1992).

[24] H.E. Stanley, P.J. Reynolds, S. Redner, and F. Family, in Real Space Renormalization, edited by T.W. Burkhardt and J.M.J. van Leeuwen (Springer, Heidelberg, 1982).

[25] B. Shapiro, J. Phys. C 11, 2829 (1978); 13, 3387 (1980).

[26] M. Napiorkowski, E.H. Hauge, and P.C. Hemmer, Phys. Lett. 72A, 193 (1979).

[27] S.L.A. de Queiroz and C.M. Chaves, Z. Phys. B 40, 99 (1980).

[28] S. Redner and P.J. Reynolds, J. Phys. A 14, 2679 (1981).

[29] F. Family, J. Phys. A 13, L325 (1980).

[30] J.A. Marqusee and J.M. Deutch, J. Chem. Phys. 75, 5179 (1981).

[31] R. Dekeyser, A. Maritan, and A.L. Stella, J. Chem. Phys. 83, 912 (1985).

[32] B. Derrida and L. De Seze, J. Phys. (Paris) 43, 475 (1982); B. Derrida and D. Stauffer, ibid. 46, 1623 (1985).

[33] A. Maritan, F. Seno, and A.L. Stella, Physica A 156, 679 (1989).

[34] K. Wu and R.M. Bradley, Phys. Rev. A 41, 6845 (1990).

[35] T. Ishinabe, J. Phys. A 18, 3181 (1985); 20, 6435 (1987).

[36] V. Privman, J. Phys. A 19, 3287 (1986).

[37] P.G. de Gennes, J. Phys. (Paris) Lett. 36, L55 (1975).

[38] J.R. Banavar, A. Maritan, and A.L. Stella, Phys. Rev. A 48, R5272 (1991).

[39] M.E. Fisher, A.J. Guttmann, and S.G. Whittington, J. Phys. A 24, 3095 (1991).

[40] G. Parisi and N. Sourlas, Phys. Rev. Lett. 46, 871 (1981). 